\title{
Introduction to the Creation and Appropriation of Knowledge Systems Minitrack
}

\author{
Stefan Smolnik \\ University of Hagen \\ stefan.smolnik@fernuni- \\ hagen.de
}

\author{
Pierre Hadaya \\ Université du Québec à \\ Montréal \\ hadaya.pierre@uqam.ca
}

\author{
W. David Holford \\ Université du Québec à \\ Montréal \\ holford.w_david@ \\ uqam.ca
}

The objective of this minitrack is to contribute to the body of knowledge that helps scholars and practitioners increase their collective understanding of

(1) how knowledge systems are planned, designed, constructed, implemented, used, evaluated, supported, upgraded, and evolved;

(2) how knowledge systems impact the context in which they are embedded; and

(3) the human behaviors reflected within and induced through both (1) and (2).

By knowledge system, we mean a system in which human participants and/or machines perform work (processes and activities) related to the creation, retention, transfer and/or application of knowledge using information, technology, and other resources to produce informational products and/or services for internal or external customers. Such systems may include, but are not limited to, knowledge management systems, decision systems, social media, expert systems, machine learning systems, and other AI systems as well as any other IT-enabled knowledge management processes.

It is the 6th year of the minitrack. We received eleven papers this year and after a rigorous review process, we accepted five papers for publication in the proceedings and presentation at the conference.

The first paper, co-authored by Nico Wunderlich and Roman Beck, looks at how the concept of a digital business strategy leads to increased organizational innovativeness and firm performance. The research results reveal that IT knowledge of business employees has a higher positive impact on organizational innovativeness in organizations giving the digital business strategy high importance, whereas the top management team IT knowledge plays a greater role when digital business strategy is given low priority.

The second paper, co-authored by Hans-Georg Fill and Felix Haerer, looks at how blockchain technologies can be applied to the domain of knowledge management. To validate the technical feasibility of the approach a first technical implementation is described and applied to a fictitious use case.

The third paper, co-authored by Dimitris Karagiannis and Robert Andrei Buchmann, proposes a deployment approach for hybrid knowledge bases using agile diagrammatic means. The proposal is based on the RDF-semantics variant of OWL and leads to a particular type of hybrid knowledge bases hosted by the GraphDB system. The approach aims for complementarity and integration, providing means of creating semantic networks that are amenable to ontology-based reasoning.

The fourth paper, co-authored by Kevin Lumbard, Ammar Abid, Christine Toh, and Matt Germonprez, focuses on understanding the types of information utilized in industry with the aim of enabling the development and sharing of methodologies that support the design of competitive products. As such, this paper proposes a framework of information archetypes utilized by designers in industry. The research results reveal two archetypes of information utilized by decision-makers within these companies during the development of new products and services.

The fifth paper, co-authored by Olivia Hornung and Stefan Smolnik, presents a systematic review of existing evidence on the role of emotions in $\mathrm{KM}$ research. The review shows that despite KM's long tradition, there is only limited evidence as to how emotions are related to KM, most of which mention emotions as motivation for KM. As a study's result, the authors identify four research opportunities to further examine certain aspects of the role of emotions in KM.

We wish to thank all of the authors who submitted work for consideration in this minitrack. We also thank the dedicated reviewers for the time and effort they invested in reviewing the papers. We believe that the accepted papers contribute to furthering our understanding on the creation and appropriation of knowledge systems. We look forward to discuss these further during our sessions in January 2018. 